\title{
Transformación Urbana y Fronteras Imaginarias en el Valle de Santiago Durante la Conquista Española (Siglo 16)
}

\author{
Daniela Derosas Contreras ${ }^{1}$
}

\begin{abstract}
Resumen
Las urbes americanas que se constituyeron a partir del siglo 16, son un espejo de la España que los conquistadores dejaban atrás. Pertenecientes a una sociedad jerarquizada y con un escaso bagaje cultural, los conquistadores impusieron su visión sobre un contexto dispar. El resultado fue la transformación forzada del paisaje y la creación de un entramado urbano disociado del entorno natural. Algunos hitos naturales sirvieron como fronteras imaginarias que separaron dos mundos que se retroalimentaban: españoles e indios. En el caso particular de Santiago de Chile, este límite estaba constituido por el río Mapocho, el cual separaba el núcleo urbano de la periferia conocida como La Chimba.
\end{abstract}

Palabras-clave: Fronteras imaginarias. Entramado urbano. Santiago de Chile. Transformación urbana.

\begin{abstract}
The American cities were formed at the beginning of the 16 century, they were a reflection of the Spanish culture that the conquerors left behind. They belonged to a hierarchical society that lacked cultural background. The conquerors transposed their vision to a different situation. The result was the forced transformation of the landscape and the creation of the different urban fabric to the natural context. Some boundary makers served as imaginary borders, they separated two worlds: Spanish and indigenous. In the particular case of Santiago-Chile, this edge was marked by the Mapocho river, which separated the urban core from the suburbs recognized as La Chimba.
\end{abstract}

Keywords: Imaginary borders. Urban fabric. Santiago-Chile. Urban transformation.

${ }^{1}$ Doctoranda en Educación, Universidad Autónoma de Madrid. Máster en Didácticas Específicas en Aulas, Museos y Espacios Naturales, Universidad Autónoma de Madrid. Máster en Formación del Profesorado para E.S.O. y Bachillerato, Universidad Autónoma de Madrid. Licenciada en Historia, Pontificia Universidad Católica de Chile. daniela.derosas@gmail.com 
El presente trabajo es el resultado de algunas inquietudes que nacen a partir del desarrollo de la tesis doctoral. Mi formación primigenia es una licenciatura en Historia, este es el punto inicial desde la cual se extiende mi visión de mundo, sin embargo en el camino la Geografía se ha erguido como una disciplina indisociable de la misma.

A lo largo del profundo estudio de la historia de América, he comprendido que esta no se entiende sin la influencia de la europea: la identidad de Latinoamérica está contaminada por diversas contribuciones entre la que destaca aquella española. Este hecho es palpable en la cultura, pero también en la fundación y construcción de las ciudades latinoamericanas, las cuales suponen una alteración (no siempre fluida) del paisaje.

La transformación del paisaje americano es un tema muy amplio, el cual se remonta previamente a la conquista española, puesto que los habitantes originarios de esas tierras ya habían incurrido en modificaciones espaciales de mayor o menor envergadura; pero fue la influencia hispana la que transformó el paisaje de forma intensa por medio de la reproducción del espacio europeo en un contexto dispar.

Los procesos de conquista y colonia se prolongaron a lo largo de tres siglos a en todo el continente americano, por lo que fue necesario limitar el período a investigar. Las transformaciones urbanas son aquellas que remiten a la fundación de ciudades, por lo tanto la investigación se acota al siglo 16, momento clave en cuanto a las primeras intervenciones españolas en América, y que marcarán una pauta de actuación que se perpetuará a lo largo tiempo.

El tema es ampliamente estudiado por la historiografía, sin embargo el aporte del presente estudio es imprimir una visión que colinda tanto con la Geografía como con la Antropología, centrándose en el tema de la nueva identidad inherente a urbanización y la percepción de fronteras imaginarias.

\section{Circunstancias históricas y geográficas}

El objetivo de la investigación es dar a conocer en qué medida las particularidades físicas de la geografía chilena han incidido en el planeamiento urbano español durante la conquista. El trazado urbano no está exento de la 
idiosincrasia del conquistador europeo, influencia que se refleja en el desarrollo de muchas ciudades de Latinoamérica, y en particular de la ciudad de Santiago de Chile; país donde existen "fronteras imaginarias".

Chile es el país más largo y estrecho del mundo, con una longitud de 4300 kilómetros de largo y un ancho promedio de 177 km. Por el norte limita con Perú, por el sur con la Antártica, al oeste con el océano Pacífico, y por el este con la Cordillera de los Andes. Está asentado sobre las placas tectónicas de nazca y sudamericana, lo que lo convierte en uno de los países más sísmicos del planeta (INSTITUTO..., 2015). Posee todos los tipos de clima (UNIVERSIDAD DE CHILE, 2015), manifestando múltiples y variados paisajes que van desde el árido desierto de Atacama, hasta las nevadas Torres del Paine en su extremo más austral.

Es esta geografía, tan hermosa y hostil, la que los nativos fueron incapaces de dominar. Pese a que gran parte de Chile estaba bajo la influencia inca, los pueblos indígenas chilenos no alcanzaron un estadio cultural desarrollado que les permitiera dominar la naturaleza, muy por el contrario, tuvieron que adaptar sus modos de vida a ella. La escaza transformación del paisaje viene acompañada de la tecnología incaica, sobre todo en lo que se refiere al aprovechamiento de los cursos de agua para regadío, pero una permuta evidente y visible es consecuencia de la creación de núcleos urbanos a partir del siglo 16.

España estaba despertando del sueño medieval entrando con pie firme en el Renacimiento, y pese a que sus ciudades son de trazado medieval (hecho evidente al contrastar los cascos antiguos con respecto a los ensanches del siglo 14), Hispanoamérica se proyecta desde sus inicios bajo las directrices del plano damero o plantilla cuadriculada. Este tipo de trazado urbano genera una ordenación racional: el corazón de la ciudad está constituido por los órganos de gobierno (Cabildo, Real Audiencia) y eclesiásticos (Catedral), que están instaurados en la Plaza Mayor o Plaza de Armas, desde la cual se trazan las calles y manzanas (o cuadras) que formarán la nueva urbe.

La fundación de ciudades en la América hispana tiene un fuerte componente simbólico: muchas localidades poseen los mismos nombres que villas españolas de donde eran oriundos sus fundadores. El homónimo respondería 
de forma consciente, a ensalzar el lugar de origen del conquistador como una manera de hacer trascender la propia patria. Pero de modo inconsciente extiende el territorio e identidad española mediante la apropiación por el nombre; lo que quiere decir que el territorio ibérico no acabaría donde la península, sino que continúa sin interrupciones ultramar. En una vertiente antropológica, el hombre crea y otorga identidad mediante el acto de nombrar; en otras palabras, algo existe o es porque se le ha dado un nombre, pero ese nombre es europeo, por tanto pertenece a Europa y absorbe parte de sus características.

La delimitación del territorio, respondería a la necesidad de control del mismo, mediante la creación de fronteras imaginarias, que conjeturan un refuerzo de la propia idiosincrasia. Funcionan como espejos, en los que un pueblo se reconoce en la alteridad con respecto a los que ya habitaban el espacio. Josep Fontana afirma que "Todos los hombres se definen a sí mismos mirándose en el espejo de 'los otros' para diferenciarse de ellos” (2000, p. 107). En el caso del hombre europeo si en un primer momento lo que diferenciaba a los unos de los otros se basaba en principios netamente religiosos, al encontrarse con los hombres del nuevo mundo, se produce un quiebre de esta concepción para dar cabida a conceptos antagónicos como "salvaje - civilizado".

La frontera se puede definir como una separación física - geográfica, o artificial; pero también posee una acepción psicológica inherente a la cultura como por ejemplo idioma o religión (TOLOSANA, 1994, p. 97). Independiente de la significación que se le conceda, una frontera crea una separación que refuerza la identidad homogeneizante de una sociedad. “(...) vemos perfilarse los dos límites de una problemática de la identidad oscilando entre el polo de una singularidad desconectada y el de una unidad globalizante poco respetuos a con las diferencias" (BERNOIST, 1981, p. 13). A la vez que distancia a un grupo humano de otro, al interior del mismo refuerza la filiación repudiando la pluralidad. 
Los límites geográficos que separan a los países, son invenciones del siglo 14, posteriores al proceso independentista, ya que la intención del imperio español era dar una visión de continuidad al vasto territorio. No obstante hubo hitos geográficos que se volvieron fronteras imaginarias como es el caso del río Bío-Bío.

Desde la zona norte de Chile hasta el río Maule, comprendido el valle central en donde se ubica la ciudad de Santiago, la dominación fue más expedita que al sur del río Bío-Bío. Esto se debe principalmente a dos motivos: por una parte el río es, aún hoy, bastante caudaloso, y sólo se podía atravesar durante los meses de verano (enero - marzo), en el invierno (julio - septiembre) era capaz de arrastrar militares y caballos. La zona estaba casi sin explorar, pese a que se logró construir algunos fuertes que por lo general eran destruidos y saqueados en las malocas (BENGOA, 2000). En segundo lugar, la zona al sur del río estaba habitada por el pueblo Mapuche, sociedad guerrera y sin una formación estatal como la entendemos hoy, sino que compuesta por muchos núcleos los cuales se reunían en tiempos de guerra. Los cronistas están de acuerdo en que era muy difícil dominarles, porque no poseían el concepto de gobierno centralizado al cual estaban acostumbrados los europeos (GOICOVICH, 2004). Ambos sucesos gestaron el mito de la incansable guerra que sostuvo Chile contra España por más de 300 años; en la práctica fueron centurias de relaciones fronterizas, en las que ambos pueblos tuvieron que aprender a convivir (MELLAFE, 2004). Así Chile mantendría su condición de Capitanías General, y el río Bío-Bío sería la primera frontera imaginaria con la que los españoles tendrían que lidiar, cambiando absolutamente la forma de relacionarse con los naturales de Chile.

\section{Algunas fuentes:}

Existen variados estudios sobre la conquista de América, la de Chile, y la fundación de la ciudad de Santiago. Ante la imposibilidad de sintetizar toda la información al respecto, y centrándome en los autores de más relevancia para la 
presente investigación, he de destacar a Armando de Ramón y su libro "Santiago de Chile. Historia de una sociedad urbana (1541-1991)." Completísima obra sobre la geografía del Valle de Santiago y su historia, que supone el punto de partida para cualquier trabajo que abarque esta temática.

El manual de Historia de Sergio Villalobos "Chile y su historia", es excelente texto de contextualización histórica que cuenta importantes sucesos de una manera sencilla y fluida. En la misma línea la obra de Rolando Mellafe, quien afronta amenamente el tema de las relaciones sociales que se dieron durante la colonia. También Ricardo Donoso, que de manera breve e incisiva permiten hacernos una idea de los diversos sucesos ocurridos en durante la fundación de Chile.

Por otra parte, he de destacar los estudios sobre el barrio de la Chimba de Gonzalo Sotomayor, el cual aporta amplitud de visión, puesto que Santiago de Chile no acabaría en el Mapocho. Con respecto al río, los estudios de Rubén Stehberg y Paulo Álvarez sobre el Mapocho Incáico iluminan el hecho de que es mucho más que un recurso natural.

Jaime Valenzuela, ha estudiado las relaciones de mestizaje y poder que se dieron en Chile, permitiéndonos mediante su trabajo conocer a esos otros habitantes que formaron el Santiago del Nuevo Extremo, en una suerte de historia desde abajo.

Hice uso de más fuentes, pero las nombradas han supuesto imprescindibles para que la investigación llegara a buen puerto.

\section{Transformación urbana y fronteras imaginarias durante la conquista de Chile}

Chile es un país de contrastes, y desde un primer acercamiento las huestes españolas fueron conscientes de este hecho. Ya en 1569, el español Alonso de Ercilla y Zúñiga lo describía en su obra "La Araucana”, poema épico sobre los principales enfrentamientos que acontecieron en la conquista del país: 
Es Chile norte sur de gran longura

Costa del nuevo mar, del Sur llamado

Tendrá del este a oeste de angostura

Cien millas, por lo más ancho tomado

Bajo del Polo Antártico en altura

De veintisiete grados, prolongado

Hasta do el mar Océano y Chileno

Mezclan sus aguas por angosto seno.

(...)

Digo que norte sur corre la tierra,

Y baña la del oeste la marina;

A la banda del este va una sierra

Que el mismo rumbo mil leguas camina

(...) $(1979$, p. 129).

La geografía no sólo impresionará a muchos cronistas españoles a lo largo del siglo 16, sino que también será determinante a la hora de abordar la conquista en todo el continente. Recordemos el ejemplo de Tenochtitlán, húmeda y cenagosa ciudad fundada en sobre el lago Texcoco, y centro político del imperio Azteca, que perjudicó la conquista española porque sus condiciones impedían la utilización de armas de fuego y armaduras. En esta ocupación fue primordial la habilidad diplomática de Hernán Cortés (FERNÁNDEZ CHRISTLIEB; GARZA MERODIO, 2011). La fundación de la ciudad de México, se cimentó sobre los antiguos restos de la capital azteca, a la usanza castellana y sin una adaptación específica para dichos terrenos. Del mismo modo, los conquistadores españoles a lo largo de todo el territorio americano incurrirían en los mismos errores perceptuales, algunas veces con resultados más afortunados que otros.

Los orígenes de la conquista de Chile se remontan al año 1536, cuando el manchego Diego de Almagro se aventura en la exploración de las tierras al sur del Perú. Pese a que había forjado una fuerte amistad con Francisco Pizarro, participando en el sometimiento del Imperio Inca, sus ganas de amasar riqueza y la ambición de poder le llevarían a romper este vínculo y buscar nuevas tierras 
en las que gobernar a sus anchas. Era un soldado reconocido por sus superiores, premiado incluso por el mismísimo Emperador Carlos V, quien de su puño y letra le anima a la conquista del inexplorado sur del Virreinato del Perú.

Los indios le informaron de la existencia de dos rutas para llegar a Chile, hasta el momento jamás surcadas por los conquistadores españoles: una primera alternativa atravesando el Desierto de Atacama, hasta llegar al Valle de Copiapó, o bien una segunda, siguiendo el camino a través de la Cordillera de los Andes a más de 4400 metros de altura; opción por la que se decidió Diego de Almagro, quien por ese entonces ya rondaba los 60 años (CORTÉS LUTZ, 2006).

La travesía fue muy penosa debido al frío y a la falta de alimentos; además, las ropas estaban completamente destrozadas, el calzado semidestruido y las herraduras de los caballos muy gastadas. Numerosos cadáveres de indios y negros quedaron en los páramos helados de la cordillera. (...) Al cabo de varios días, pudieron finalmente bajar al valle de Copiapó. (...) Almagro y sus hombres comprendieron que Chile no era el país con que habían soñado y que la abundancia de oro había sido un simple engaño de los incas para alejarlos del Perú (VILLALOBOS, 2005, p. 62).

Vencido por las vicisitudes, Almagro decide volver al virreinato, $\mathrm{y}$ tres años más tarde, en 1539, el extremeño Pedro de Valdivia asume la misión optando por la ruta que atraviesa el Desierto de Atacama, la cual era utilizada por los mensajeros Incas, y por ende más fiable. La expedición apenas alcanzaba los 10 o 12 soldados (entre los cuales destaca la figura femenina de Inés de Suarez), y un pequeño contingente de yanaconas; afortunadamente algunos militares rezagados se le sumaron en el camino, alcanzando un ejército de 152 hombres (VILLALOBOS, 2005).

El viaje fue duro, desde el inicio la geografía jugó un papel determinante en la conquista de Chile. A la enorme distancia a través del desierto de Atacama, se le añadió la escases de agua. Una vez llegados al Valle de Copiapó se abasteció en esta fértil tierra, para continuar hasta el valle central. 
El 12 de febrero de 1541, Valdivia procedió a fundar la ciudad, que quedó situada al poniente del cerro Huelén (Santa Lucía), entre el río Mapocho y un brazo seco en la actual Alameda (VILLALOBOS, 2005, p. 66). Era la ciudad de Santiago, la que se convertiría en la capital del nuevo reino y que estaría fundada en una cuenca muy fértil y atravesada por un caudaloso río.

Pedro de Valdivia sería el primero en informar en sus cartas dirigidas al emperador Carlos V, sobre el territorio chileno, tierras que se diferenciaban por mucho con respecto a su Extremadura natal:

Certifico a V.M. que después de que las Indias se comenzaron a descobrir, hasta hoy, no se ha descubierto tal tierra a V.M.: es mas poblada que la Nueva España, muy sana, fertilísima e apacible, de muy lindo temple, riquísima de minas de oro, que en ninguna parte se ha dado cata que no se saque, abundante de gente, ganado e mantenimiento, gran noticia, muy cerca, de cantidad de oro sobre la tierra, y en ella no hay otra falta sino es de españoles y caballos (DONOSO, 1970, p. 8).

De sus palabras se infiere que Pedro de Valdivia veía en Santiago la oportunidad de una capital riquísima, acaso comparable al Perú.

La cuenca en donde Valdivia fundaría la ciudad de Santiago de Chile (La latitud y la longitud de Santiago, la capital de Chile son $33^{\circ} 27$ S y $70^{\circ}$ 40 de longitud oeste, respectivamente ${ }^{2}$ ), en el centro del (actual) país, está en la zona llamada depresión intermedia, la cual se encuentra entre dos cadenas montañosas de gran envergadura: la cordillera de los Andes y la cordillera de la Costa, en la que además abundan los volcanes (DE RAMÓN, 1992). Al mismo tiempo, dentro de la cuenca existen cerros aislados, como por ejemplo el Cerro Huelén (actualmente llamado Santa Lucía), el cual se localiza enfrente del río Mapocho. No sería hasta entrado el siglo 17, concretamente el año 1647, cuando el llamado "terremoto magno", desplomara la ciudad y dejara en evidencia que la geografía de Chile era tan indomable como sus nativos (AMUNATEGUI, 1882).

2 Disponible en: <http://espanol.mapsofworld.com/continentes/sur-america/chile/latitud-longitud-de-chile.html>. Acceso en: 2 nov. 2015. 
La cuenca de Santiago es atravesada por varias corrientes fluviales, todas las cuales convergen hacia el río Maipo (...) especialmente el río Mapocho que cruza la actual ciudad de Santiago (...). (De Ramón, 1992, p.15) Con sus $110 \mathrm{~km}$ de largo y una cuenca de $4.230 \mathrm{~km} 2$, el río Mapocho baja torrentosamente desde una altura de $1.159 \mathrm{msnm}$, desembocando en el río Maipo a $252 \mathrm{msnm}$. Configura y da soporte al valle que cobija a la ciudad de Santiago (...) (KATZ; REID; ANDRADE, 2009, p. 56).

Es un afluente que desde los inicios sufrió múltiples transformaciones, y pese a que era utilizado desde antaño por los pueblos nativos para el regadío de las fértiles tierras del valle, se podría afirmar que con el tiempo ha perdido su condición de "río" propiamente tal y en el sentido literal para transformarse en una frontera imaginaria que separaba a españoles e indios. A partir del siglo 18 (KATZ; REID; ANDRADE, 2009) pasaría a ser el desagüe de las aguas negras de la ciudad, anulando su uso natural, y creando una escisión social mayor si cabe entre ambas riberas. En el imaginario colectivo es un río que jamás ha sido tal.

La ocupación del valle se remonta mucho antes a la llegada de las huestes españolas. La dominación del imperio Inca se extendía alrededor de 4300 kilómetros de largo ${ }^{3}$, desde el norte de Ecuador hasta el valle de Maule en Chile, supremacía detenida por la presencia del ya mencionado río Bío-Bío, barrera natural e imaginaria que ni siquiera los conquistadores pudieron superar. El río Mapocho también funcionó como frontera imaginaria en Santiago, separando a los estamentos de la sociedad, su localización fue propicia para definir el límite de la ciudad.

La dominación de la zona central fue mucho más expedita que al sur de Chile. Al respecto existen dos teorías del poblamiento del Santiago precolombino:

Stehberg (1976c), a partir de estudios realizados en la fortaleza de Chena (20 km al sur de Santiago) y del mapeo de los principales sitios con vestigios incas conocidos hacia esa fecha, postuló un modelo de la ocupación

Disponible en: $<$ http://www.mardechile.cl/index.php?option=com_content\&task=view\&id=477 \&Itemid=31> . Acceso en: 28 oct. 2015. 
Tawantinsuyu del valle del Mapocho-Maipo, basado en la existencia de una instalación urbana principal. Esta centró funcionó como "centro administrativo y eventual proveedor de abastecimiento a las tropas encargadas de la conquista...La existencia de un importante asentamiento inca en las márgenes del río Mapocho (Santiago) con un avanzado sistema de regadío y una población indígena abundante, convenció rápidamente a Pedro de Valdivia y sus hombres a establecerse en la zona y fundar la ciudad de Santiago de Nueva Extremadura (STEHBERG; SOTOMAYOR, 2012, p. 86).

Esta hipótesis de la dominación del imperio inca sobre la zona de la cuenca, es la más difundida hasta hoy por la historiografía chilena. Esta teoría avalaría que la facilidad con que Valdivia se hizo del territorio central (en contraste con la dificultad del sur) se debe a que los naturales que habitaban Santiago estarían acostumbrados al sometimiento, al igual que ocurrió (obviando salvedades) en los grandes imperios. Sin embargo

La propuesta de Stehberg (1976c) fue rebatida poco después por Silva (197778) que, luego de analizar distintas fuentes documentales, concluyó que la dominación de la cuenca de Santiago fue tenue, incompleta y tardía (...) Basado en la divergencia de los cronistas respecto a la delimitación de la frontera sur del imperio, Silva (1977-78) postuló una situación de ambigüedad respecto a la presencia de la estructura imperial al sur del Aconcagua, donde se podría "demostrar que aún en los lindes de Santiago no se habían impuesto las leyes y costumbres incaicas" y donde los testimonios documentales dan la impresión que no se había impuesto la tradicional estructura económica y social del imperio (STEHBERG; SOTOMAYOR, 2012, p. 86).

Pueblos que no se sentían parte de una misma unidad, como en el caso de los Mapuches, no se reunían en situaciones de guerra ni tenían un estadio cultural avanzado, por lo que la supremacía española sería fructífera.

Con todo, y sea cual sea la conjetura por la cual se incline, existen evidencias de asentamiento en la zona y disfrute del río Mapocho. Esto, más la posición estratégica de Santiago con respecto al sur tras el desastre de Curalaba (ÁLVAREZ, 2011), vendría a explicar por qué la capital de Chile se fundó en este valle y no más al norte en la ya fundada ciudad de La Serena. 
Pedro de Valdivia hace lo propio, llamando a Santiago de Chile, "Santiago del Nuevo Extremo” (DE RAMÓN, 2001, p. 23), la capital es la nueva Extremadura.

El trazado de la ciudad hispánica en las Américas continúa, allende el Atlántico, la tradición de las fundaciones medievales, desde que el plano en cuadrícula aparece en el trazado de Santo Domingo de 1502, hasta que en las ordenanzas de la tercera década del siglo XVI el antiguo esquema imperial se imbuye de las nuevas enseñanzas del Renacimiento (PALM, 1951, p. 59).

La fundación de la ciudad de Santiago concuerda con el último tercio del siglo 16, por lo que fue proyectada el estricto rigor del plano damero o plantilla cuadriculada. Este tipo de traza urbana se caracteriza por tener una plaza central o plaza mayor en la cual figuran el Cabildo, la Real Audiencia, y la Catedral, desde la cual se trazan las calles y manzanas que conforman la urbe. Esto tiene un sentido simbólico, porque el corazón de la urbe está compuesto por los poderes fácticos más importantes: Estado e iglesia, los cuales se disputaban la supremacía social.

Además de albergar los órganos de gobierno, y constituir el centro de la ciudad, la plaza mayor recibía el mercado de abastos y principales actos, del mismo modo que ocurría en Europa. Eso se debe fundamentalmente a que:

(...) la urbe indiana es la expresión de un sistema políticamente integrado, disgregado territorialmente, pero que presenta un desarrollo morfológico común, una funcionalidad productiva básica, determinada por la relación comercial con el virreinato o la metrópoli, y la necesidad de delimitar estamentalmente la espacialidad; todo con el fin de asegurar la reproducción económica y social del proyecto hispano-colonial (GÓMEZ ALCORTA; PRADO BERLIEN; OCARANZA BOSIO, 2014, p. 242).

Es decir que antes de siquiera fundarse, las ciudades latinoamericanas ya estaban pensadas para equipararse a las españolas. Es así como tendrían un orden preestablecido que regía desde el gobierno central en Europa, en todos 
los ámbitos: debía estar rigurosamente ordenada para cumplir sus objetivos productivos, pero también ordenar y asegurar el control de todos los estamentos que componen una sociedad jerarquizada.

Santiago tenía alrededor de 126 manzanas trazadas desde la plaza, y que conforme el territorio se acercaba al río Mapocho, el territorio se dividía en grandes fundos (DE RAMÓN, 1992). Puede que el río supusiera el límite natural de la ciudad, mas existía una población que debía ser reubicada: los indios. Mientras que los sectores acomodados que configuraban la aristocracia vivían cercanos a la Plaza de Mayor (Plaza de Armas) al extremo sur de la rivera del Mapocho, el extremo norte se reservó para "los otros", esos que no cabían dentro del concepto hispano de sociedad.

La guerra de ocho siglos que sostuvieron los reinos de España para arrojar a los invasores árabes, influyó profundamente en las características de la sociedad hispana. (...) La honra se basaba en la pureza de la sangre, sin mezcla (...) Los bienes económicos eran inseparables de la nobleza y por eso los modestos hidalgos penaban por encontrar fortuna. (...) Al noble y al hidalgo les estaba vedado el trabajo manual o cualquier tarea propia de villanos (...) (VILLALOBOS, 2005, p.77-78).

A América fueron los hidalgos en busca de oportunidades con el deseo de convertirse en aristócratas, por eso relegaron el trabajo agrícola y artesano en indios y ladinos. Es relevante recordar que el premio por la conquista era tierras y encomiendas, por lo que según Armando de Ramón, en Chile, como en el resto de América Latina, se instauró un "régimen señorial castellano" (DE RAMÓN, 2001), en el que un pequeño porcentaje de la sociedad se beneficiaba económicamente y ostentaban los principales cargos públicos.

El valle del Mapocho ya estaba poblado de indígenas a la llegada de las huestes (STHEBERG; SOTOMAYOR, 2012), pero a estos se sumaron también indios yanaconas, y ladinos como se explica ampliamente en el trabajo de Jaime Valenzuela (2014). En una sociedad estamental como lo fue la colonial, mucho más a mediados del siglo 16, no podían cohabitar en el mismo espacio que los españoles. En una sociedad donde todo tiene un orden simbólico, el 
espacio debía delimitarse, y es por eso que se les reubicó en extremo norte del río Mapocho, en el llamado barrio de la Chimba. El río Mapocho pasa a ser una frontera imaginaria que separa dos componentes de una misma sociedad.

La palabra Chimba es de origen quechua, lo que habla de un conocimiento y disfrute por parte de los incas de este espacio. En 1905, el cronista Tomás THAYER OJEDA describía que:

[...] por el año de 1560 principió a formarse sobre la ribera norte del Mapocho un barrio pobre, habitado únicamente por indígenas i yanaconas o indios del Perú que trajeron consigo los conquistadores para su servicio i denominado La Chimba, por corrupcion de chimpa, palabra quichua que significa del otro lado i con que lo designaron los naturales por estar separado de la ciudad por el mencionado rio (QUILODRÁN RUBIO; SAHADY VILLANUEVA; BRAVO SÁNCHEZ, 2014, p. 139).

Es interesante notar, que pese a que este territorio era parte fundamental de la ciudad, no se le otorgó nombre europeo. La relegación de La Chimba respondería a un afán de ocultar todo aquello que no está rigurosamente reglado o que no coincide con la imagen que España quiere proyectar en América.

Este espacio era utilizado para el cultivo y abastecimiento agrícola y ganadero del lado sur, lo que continuó siendo así luego de la fundación de la ciudad. Los nuevos habitantes de la Chimba fueron quienes se dedicaron a oficios manuales y serviles para los que vivían en la ribera sur. Eran dos grupos humanos muy distintos, que se necesitaban mutuamente, pero que estaban separados por la frontera imaginaria del río Mapocho.

La población de la Chimba quedó reflejada en algunas actas testamentares que dan luces sobre el tipo de pobladores que habitaban esta zona: indios libres, artesanos, pero también españoles empobrecidos que habían perdido su posición de poder. A su vez, el tipo de urbanización pareciera ser espontánea, al contrario del riguroso orden del otro lado del río (ÁLVAREZ, 2011). 
Esta frontera imaginaria dividió dos tierras que seguían su propio ritmo de vida. La Chimba era una ciudad por sí misma que: “(...) terminó por formar un espacio; a) popular y marginado; b) caracterizado por el trabajo artesanal y c) étnicamente heterogéneo" (ÁLVAREZ, 2011, p. 26).

A fines del siglo 16 se construyeron el Monasterio de las Agustinas, y el Convento de Santo Domingo, justamente en el límite de la ciudad por la ribera sur, antes del río Mapocho (DE RAMÓN, 1992). Pareciera que estas grandes edificaciones intentaran ocultar que al otro lado del curso de agua existe otra ciudad, caótica y poblada por marginales. Como si Santiago del Nuevo Extremo tuviera desde sus inicios, algo que no sabe donde esconder.

\section{Conclusiones}

La transformación del paisaje a lo largo del continente americano no comenzó con los conquistadores españoles, sin embargo ellos potenciaron esta situación de forma consciente y la mayoría de las veces pautada.

Existen múltiples ejemplos de cómo el español intentó dominar a América y sus habitantes por medio de la urbanización y hacerla suya a través los topónimos. A ese continente indómito y caótico que no había sido salvado de la barbarie era necesario corregirlo, reglarlo y homogeneizarlo; sin embargo, el único referente de ciudad muchos de los conquistadores españoles tendrían para el 1500 era el de sus propias villas y pueblos. Las ciudades hispanoamericanas no se construyeron pensando en América, sino en Europa, puesto que no existe una real adaptación al medio geográfico, más bien pareciera ser una reproducción fiel de la península ibérica.

Esta urbanización a la fuerza, va acompañada de brindar una nueva identidad mediante la otorgación de un nuevo nombre a la usanza española. Renombrar los sitios fundados como villas de España para hacer extensivo un territorio ultramarino se volvió una costumbre que incluso contagió a los indígenas que con el bautismo recibían un nombre "occidentalizado", como si ello les privara por fin de la barbarie y la ignorancia. 
Además de desafiar tierras desconocidas para las cuales la mayor parte de las veces no tenían las herramientas y armas adecuadas, tuvieron que enfrentarse a nuevas sociedades y a sus agentes sociales. Lidiar con lo desconocido les llevó a crear fronteras imaginarias que separaban una población de otra, como forma de poder cohabitar en paz.

En el caso de Santiago del Nuevo Extremo, ciudad que al igual que La Serena, por su toponimia remite a la Extremadura natal de Pedro de Valdivia, el río Mapocho funcionó como un hito natural que separaba a dos sociedades que no podían vivir la una sin la otra, pero que buscaron reconocerse en la alteridad, como si ese río funcionara a modo de espejo deformante.

Para concluir, gran parte de la idiosincrasia chilena, y principalmente en Santiago, se debe a la herencia colonial. Todavía se puede apreciar una escisión entre estamentos de la sociedad, pese a que han hecho una permuta en cuanto a localización, y es la "aristocracia" quien habita en la periferia cordillerana por encima y lejos del contaminado centro.

¿Será que las fronteras imaginarias nunca desaparecen, y caminan al ritmo de la transformación urbana?

\section{Referencias}

ÁLVAREZ, Paulo. La Chimba del Valle del Mapocho: historia de una alteridad en construcción. (Siglos XV-XIX). Espacios. Revista de Geografía, n. 1, p. 19-42, 2011. DOI: http://bibliotecadigital.academia.cl/handle/123456789/3003

AMUNATEGUI, Miguel Luis. El terremoto del 13 de mayo de 1647. Santiago: Editor Rafael Jover, 1882.

BENGOA, José. Historia del pueblo mapuche. (Siglo XIX y XX). Santiago: Lom Ediciones, 2000.

BERNOIST, Jean Marie. Las facetas de la identidad. In: LEVI-STRAUSS, Claude. La identidad: seminario interdisciplinario. Barcelona: Ediciones Petrel, 1981.

CORTÉS LUTZ, Guillermo. Análisis crítico de la entrada del adelantado Diego de Almagro en Chile en 1536. Revista Clio: History and History teaching, vol. 32, 2006. Disponible en: <http://clio.rediris.es/numero032.htm>. 
DE RAMÓN, Armando. Breve historia de Chile. Desde la invasión incaica hasta nuestros días (1500-2000). Buenos Aires: Ed. Biblos, 2001.

. Santiago de Chile (1541-1991): Historia de una sociedad urbana. Madrid: Editorial Mapfre, 1992.

DONOSO, Ricardo. Breve historia de Chile. Buenos Aires: Editorial Eudeba, 1970.

ERCILLA Y ZÚÑIGA, Alonso. La araucana. Madrid: Editorial Castalia, 1979. Volumen I.

FERNÁNDEZ CHRISTLIEB, Federico; GARZA MERODIO, Gustavo. Cultura y territorialidad en la ocupación de un mismo espacio: Mexico, Tenochtitlan y la Ciudad de Mexico en el siglo XVI. Revista GeoTropico, v. 5 (2), p. 53-64, 2011. DOI: http://www. geotropico.org/NS_5_2_Fernandez-Garza.pdf

FONTANA, Josep. Europa ante el espejo. Barcelona: Editorial Crítica, 2000.

GOICOVICH, Francis. Reevaluación Etnohistórica de las Ayllareguas Reche-Mapuches. Tomo I Actas $5^{\circ}$ Congreso Chileno de Antropología. 2004. p. 558-565. DOI: https://www. aacademica.org/v.congreso.chileno.de.antropologia/73

GÓMEZ ALCORTA, Alfredo; PRADO BERLIEN, Claudia; OCARANZA BOSIO, Francisco. Construcción del espacio urbano y modelación social desde la "ciudad letrada": Santiago, Chile (Siglos XVI-XVIII). Historelo: Revista de Historia Regional y Local, v. 6, n. 12, p. 237-279, 2014.

INSTITUTO GEOGRÁFICO MILITAR DE CHILE. 2015. Disponible en: <http://200.27.184.149/IGMChile/>. Acceso en: 28 oct. 2015.

KATZ, Carolina; REID, Paul; ANDRADE, Martín. Tres visiones sobre el río Mapocho. Revista ARQ, n. 72, p. 56-59, 2009.

MELLAFE, Rolando. Historia social de Chile y América. Santiago: Editorial Universitaria, 2004.

PALM, Erwin Walter. Tenochtitlan y la ciudad ideal de Dürer. Revista Journal de la Société des Américanistes, tomo 40, p. 59-66, 1951. DOI: http://www.persee.fr/doc/ jsa_0037-9174_1951_num_40_1_2542

QUILODRÁN RUBIO, Carolina; SAHADY VILLANUEVA, Antonio; BRAVO SÁNCHEZ, José. Estudio del norte de Santiago mediante cartografía histórica: la Chimba entre los siglos XVI y XIX. Revista Papeles de Geografía, v. 59-60, p. 137-156, 2014.

STEHBERG, Ruben; SOTOMAYOR, Gonzalo. Mapocho incáico. Boletín del Museo Nacional de Historia Natural, v. 61, p. 85-149, 2012. 
THAYER OJEDA, Tomás. Santiago durante el siglo XVI: Constitución de la propiedad urbana i noticias biográficas de sus primeros pobladores. In: QUILODRÁN RUBIO, Carolina; SAHADY VILLANUEVA, Antonio; BRAVO SÁNCHEZ, José. Estudio del norte de Santiago mediante cartografía histórica: la Chimba entre los siglos XVI y XIX. Revista Papeles de Geografía, v. 59-60, p. 137-156, 2014.

TOLOSANA, Carmelo Lisón. Antropología de la frontera. Revista Antropología Social, v. 3, p. 75-103, 1994. DOI: http://revistas.ucm.es/index.php/RASO/article/view/ RASO9494110075A/10460

UNIVERSIDAD DE CHILE. 2015. Disponible en: <http://www.uchile.cl/portal/presentacion/la-u-y-chile/acerca-de-chile/8086/climas-de-chile> . Acceso en: 28 oct. 2015.

VALENZUELA, Jaime. Indios urbanos, inmigraciones, alteridad y ladinización en Santiago de Chile (siglos XVI y XVII). Revista Historia Crítica, n. 53, p. 13-34, 2014. VILLALOBOS, Sergio. Chile y su historia. Santiago: Editorial Universitaria, 2005.

\section{Agradecimientos}

Gracias especialmente a Alfonso García de la Vega, mi tutor y maestro, por creer en mí y enseñarme a mirar la Historia con ojos de Geógrafo; y Vignia González su traducción y amistad.

Recebido em: 5/6/2016

Aceito em: 10/11/2016 